\title{
NORM ESTIMATES FOR BESSEL-RIESZ OPERATORS ON GENERALIZED MORREY SPACES
}

\author{
Mochammad Idris, Hendra Gunawan, Bandung, \\ ERIDANI, Surabaya \\ Received May 11, 2017. First published March 7, 2018. \\ Communicated by Dagmar Medková
}

\begin{abstract}
We revisit the properties of Bessel-Riesz operators and present a different proof of the boundedness of these operators on generalized Morrey spaces. We also obtain an estimate for the norm of these operators on generalized Morrey spaces in terms of the norm of their kernels on an associated Morrey space. As a consequence of our results, we reprove the boundedness of fractional integral operators on generalized Morrey spaces, especially of exponent 1 , and obtain a new estimate for their norm.
\end{abstract}

Keywords: Bessel-Riesz operator; fractional integral operator; generalized Morrey space $M S C$ 2010: 42B20, 26A33, 42B25, 26D10

\section{INTRODUCTION}

Integral operators such as maximal operators and fractional integral operators have been studied extensively in the last four decades. Here we are interested in BesselRiesz operators, which are related to fractional integral operators. Let $0<\alpha<n$ and $\gamma \geqslant 0$. The operator $I_{\alpha, \gamma}$ which maps every $f \in L_{\text {loc }}^{p}\left(\mathbb{R}^{n}\right), 1 \leqslant p<\infty$, to

$$
I_{\alpha, \gamma} f(x):=\int_{\mathbb{R}^{n}} K_{\alpha, \gamma}(x-y) f(y) \mathrm{d} y=K_{\alpha, \gamma} * f(x), \quad x \in \mathbb{R}^{n},
$$

where $K_{\alpha, \gamma}(x):=|x|^{\alpha-n}(1+|x|)^{-\gamma}$, is called Bessel-Riesz operator, and the kernel $K_{\alpha, \gamma}$ is called Bessel-Riesz kernel. The boundedness of these operators on Morrey spaces and on generalized Morrey spaces was studied in [8] and [9].

The first and second authors are supported by ITB Research \& Innovation Program 2016. 